\title{
دور التعليم الرقمي للتربية الفنية في مواجهة الأثار السلبية لجائحة كورنا
}

The role of art education digital education in facing the negative effects of the Kurna pandemic

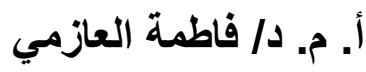

أستاذ مساعد كلية التربية الأساسية قسم التربية الفنية

\section{Assist. Prof. Dr. Fatema Al-azimy \\ Assistant Professor of the College of Basic Education, Department of Art Education fma13@my.fsu.edu}

\begin{abstract}
ملخص البحث
في فترة مرور الوطن العربي بجائحة كرونا الكل لمس الدور البارز الذي لعبه الفن و الفنانين في هذه الفترة على المستوى الفي

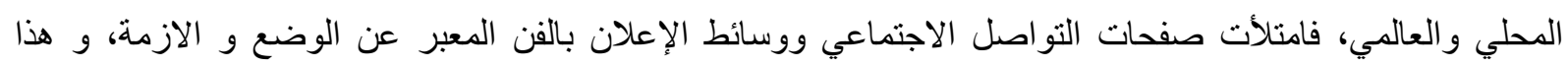

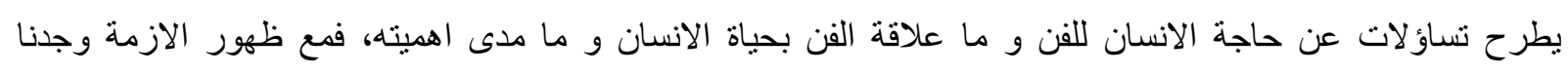

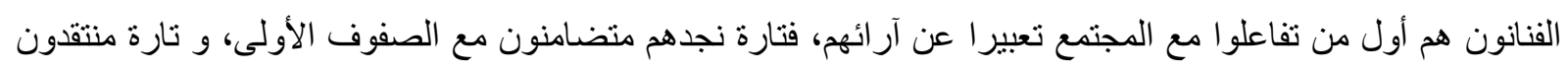

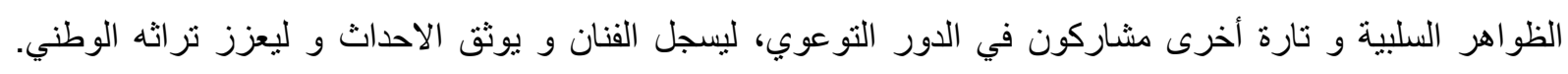
فيتجلى بوضوح أهمية الفن من الناحية التربوية و النفسية و الإنسانية.

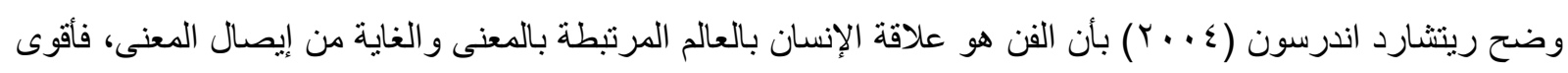
ميزة للفن هي علاقته بحياتتا. فلذلك، يجب تسليط الضوء على أهمية تعليم الفن في حياتنا اليومية، وللمجتمع ككل بطريقة معاصرة.

واستهف البحث دراسة تحليل الأطر النظرية لدفاهيم التعليم الرقمي للتربية الفنية وعلاقته بالنظريات العلمية والفنية المعاصرة وكذلك دوره التعليم الرقمي للتربية الفنية في مواجهة الأثار السلبية لجائحة كورونا. وتناول البحث دراسة مجمو عة نقاط هامة مرتبطة بموضوع البحث وهي: دراسة لتطبيقات التعليم الرقمي في التربية الفنية التي تساعد المتعلم في التحصيل عن بعد كما تناول البحث أيضا استخدامات هذه التطبيقات الرقمية في التغلب على سلبيات

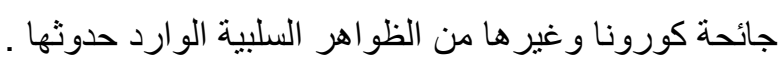

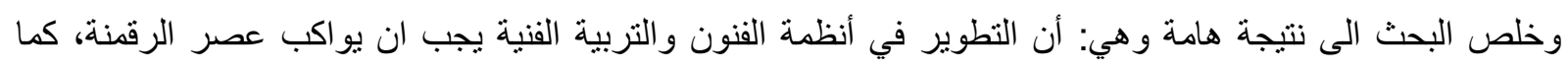
توصل البحث الى ان هنالك آثار سلبية لجائحة كورونا على تعليم الفن و التربية الفنية. الكلمات المفتاحية: التعليم الرقمي , مواجهة, الأثار السلبية, جائحة كورنا
\end{abstract}

\begin{abstract}
Through the Corona pandemic period in the Arab world, everyone touched the prominent role that the art and artists played in this period at the local and global levels. This raises questions of the human need for art, how art is related to our life, and why it is vital?

We can see clearly with the rise of the crisis. The artists were the first who interact with the society expressing their views: solidarizing with the frontline, Critiquing negative attitudes, and educating their society. Accordingly, the artists document the events and reinforce their social heritage. Therefore, the significance of art is interrelated clearly from the educational, psychological, and humanitarian aspects.
\end{abstract}


Richard Anderson (2004) contended that art has a culturally significant meaning, as human's relation to the world is linked to meaning and the purpose of communicating meaning. The most powerful feature of art is its relation to our life. Therefore, my intention is to illuminate the usefulness of art in our everyday lives, and for society at large.

The research aims to study and analyze the theoretical frameworks for digital education concepts in art education confronting the negative effects of the Corona pandemic. The research covered important points related to the research study. For instance: a study of the applications of digital education in art education that supports the student through distance learning. Also, the research discussed the uses of these digital applications to overcome the negative aspects of the Corona pandemic or any other struggles that could occur.

The research guided to an important conclusion: that development in the arts and art education systems must keep pace with the era of digitization and the research also found that there are negative effects of the Corona pandemic on teaching art and art education.

\section{Keywords:}

Digital education, facing, negative effects, pandemic Corona

في بداية عام 2020 غزى فيروس كرونا العالم ليشل الحركة الاجتماعية و الاقتصادية و السياسية، لتعيش المجتمعات بعزلة و تباعد اجتماعي، ففرض الاغلاق و العزل و التباعد، فوجد الانسان نفسه محاصر في بيته ليواجه نفسه و افكاره مع هذه التجربة، في هذه الفترة وجدنا تفاعل ملحوظ مع الفن، فاكتسح الفن ميادين الاعلام و وسائل التو اصل الاجتماعي، لئه



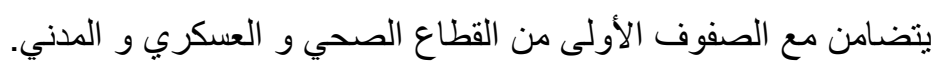
و مع فرض الاغلاق و إلزام الافراد في المجتمع بقوانين الحجر و العزل، تعطلت كل الاعمال ما عدا الفن فهي الوسيلة



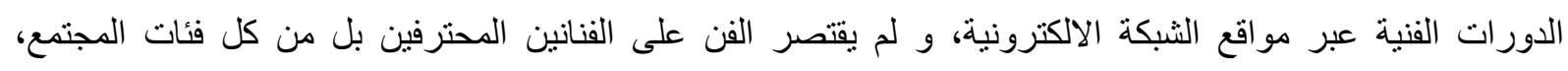
فتوجهت الاسر و الافراد بطريقة عفوية بإنتغال فراغهم بالفن للخروج من هذه الضغوطات النفسية التي أفرزتها هذه

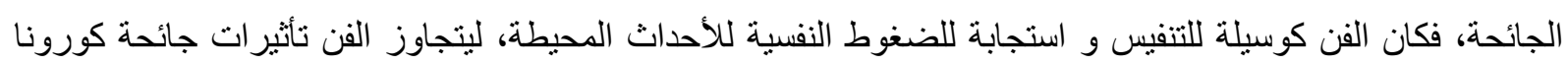
فكان بمثابة نافذة مفتوحة للخروج من الضيق لتطل على العالم الخارجي. فلم ينعزل الفنان بل هو من أول من تفاعل و تضامن مع محيطه، ليوثق الاحداث في سجلات التاريخ، فالفنان من طبعه يمنلك إحساس ملح بأهية التعبير عن آرائه لينسجم مع مجتمعه و يعزز وطنيته، و من هنا ينطلق التساؤل، ما هي أهمية الفن في حياة الانسان؟ هل له دور ثانوي ام حيوي؟ و اذا كان كذلك، كيف نفعل دور الفن في مجال التربية و التعليح.

تضامنا مع ازمة كرونا العالمية يسعى البحث الى إيضاح دور التربية الفنية في مواجهة الأثار النفسية و السلبية لجائحة

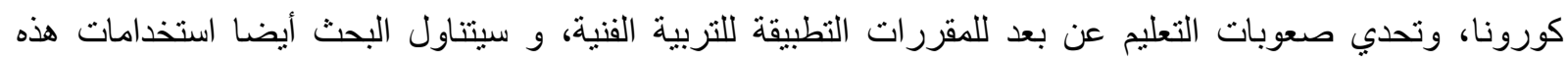

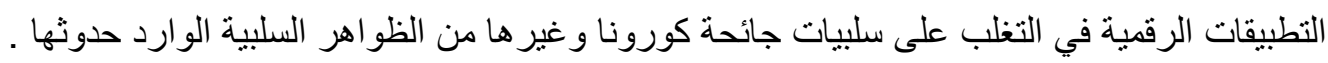


استهدف البحث دراسة تحليل الأطر النظرية لمفاهيم التعليم الرقمي للتربية الفنية وعلاقته بالنظريات العلمية والفنية المعاصرة وكذللك دور التعليم الرقمي للتربية الفنية في مواجهة الأثار السلبية لجائحة كورونا. وتناول البحث دراسة مجمو عة نقاط هامة مرتبطة بموضوع البحث وهي: دراسة لتطبيقات التعليم الرقمي في التربية الفنية التي تساعد المتعلم في التحصيل عن بعد كما تتاول البحث أيضا استخدامات هذه التطبيقات الرقمية في التغلب على سلبيات

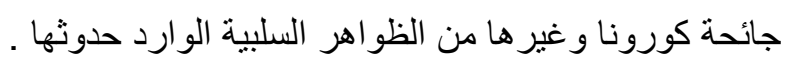

قد يفيد البحث الحالي فيما يلي: • التأكيد على الدور الفعال للتربية الفنية لتخفيف الآثار النفسية في وقت الازمات. • تعزيز الوطنية و روح القيادة من خلال اندماج الفرد بالمجتمع و حل قضاياه. تأكيد الدور التوعوي و التضامني للفن في المجتمع. التعرف على أساليب التربوية الحديثة في التعليم التطبيقي عن بعد.

تم تطبيق البحث على أجمالي 70 طالبه منهن(40 طالبة) تصوير في المستوى1 (30 طالبة) تصوير في المستوى2 في

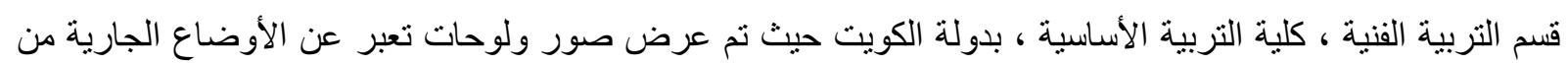
الآثار النفسية و الاجتماعية لازمة كرونا. حيث كُلفت الطالبات بعمل موضوع يتحدث عن ازمة جائحة كرونا وتعبر عن الأوضاع الجارية في الكويت سواء كانت

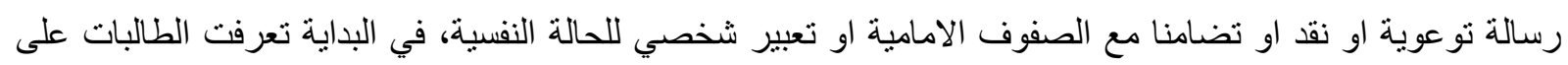
بعض الفنانين المحترفين في الكويت و ناقشنا تفاعلهم و لوحاتهم التي عبرت عن الازمة، و درست الطالبات من خلالها

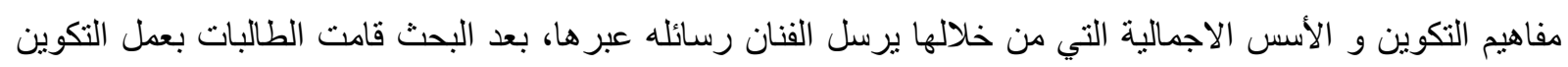

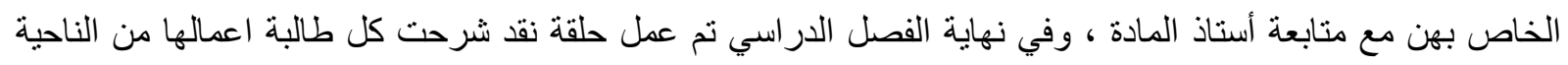

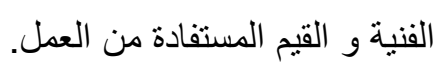

منهج البحث : البح : و أتبع البحث المنهج الوصفي التحليلي فيما يخص الإطار النظري ، كما يتبع المنهج التجريبي فيما يخص الجانب العملي للبحث .

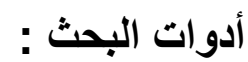
تم تصميم استيبان و مقابلات شخصية لاستطلاع ر أي الطالبات لمعرفة الى أي مدي استفادة الطالبات من اهداف الدرس.

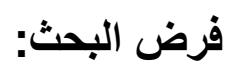
امكان معالجة الآثار السلبية للازمات و الضغوطات من خلال التربية الفنبة من خلال التعبير عن الاوضاع و التفاعل معها بإيجابية .

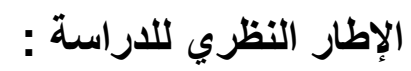
لقد برز دور الفن من خلال الازمة جائحة كوروناو تجاوب الفنانون على المستوي المحلي و العالمي في مواجهة الازمة،

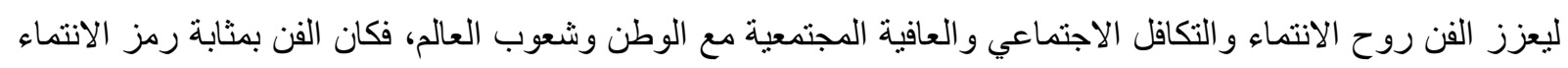


الوطني و الإنساني و لغة عالمية موحدة لتضامن الثعوب، لتكون الوسيلة الوحيدة لتنتصر الإنسانية في هذه المعركة. مما

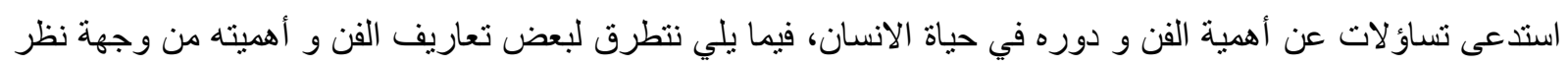
الباحثين و المفكرين.

\section{ما هو الفن:}

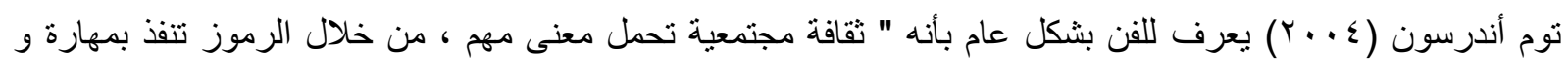
اتقان في وسط حسي مؤثر. وعرف ريتشارد اندرسون (ع . . ب) الفن " بأن علاقة الإنسان بالعالم المرتبطة بالمعنى و الغاية من إيصال المعنى، فنرى ذلك في العديد من المجتمعات الذين استثمروا الفن كوسيلة لتوثيق حكمتهم أو رؤاهم العميقة، الحقيقة المبنية على الخبرة البشرية التي عادة تكون لها قبول جماعي.

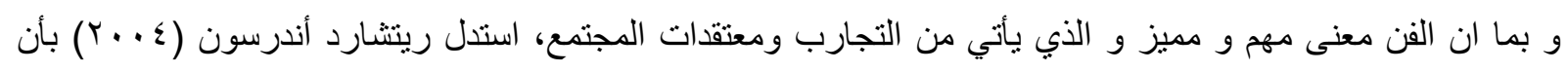

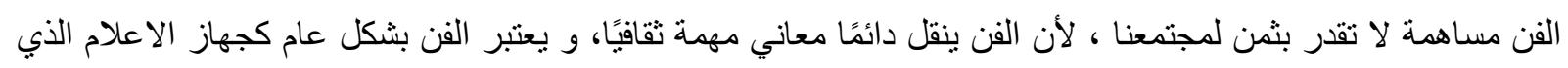

يمثل قيم المجتمع كمظاهر للحقيقة. و فسر أندرسون وميلبرانت (2005) أن الفن حاجة بيولوجية ونفسية ، فالإنسان يسعى إلى صنع معاني من خلال الأثكال الجمالية ليؤثر في محيطه كنوع من التو اصل، فاستخدمت المجتمعات و الثقافات الفن لبناء معنى لمساعدة أنفسهم

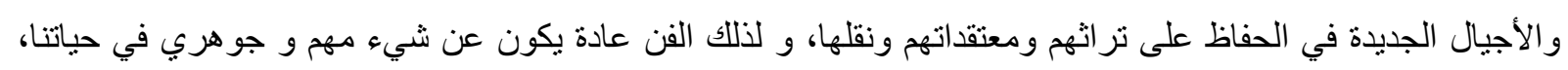



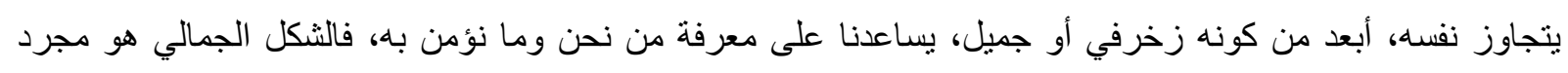


تخبرنا شييًا عن التجربة الإنسانية. نستنتج ان جوهر الفن هو خلق معنى، إنه وجهات نظرنا في الحياة وقصصنا وآراعنا، لغة الفن هي طريقتنا البشرية للتواصل عبر الأجيال والحفاظ على تراثنا وهويتنا، يعمل الفن كوسيلة للتواصل، وهو أساس المجتمع الديمقراطي (Dewey, 1934)، لذلك، الفن لا يمثل الفرد فقط، بل يمثل القيم المشتركة للمجتمع. وبالتالي، نحن بحاجة إلى استعادة

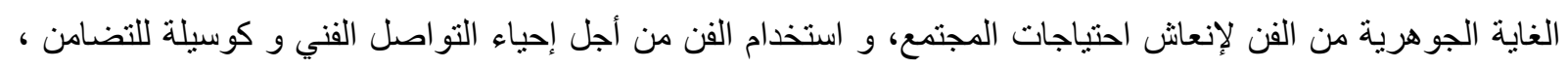

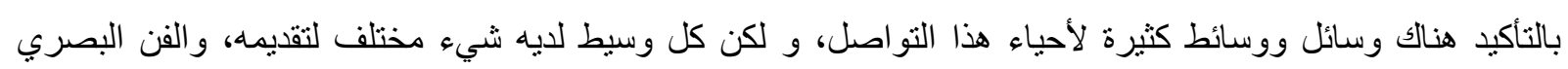



\section{التربية الفتية وتعزيز الروح القيادية و والوطنية:}

يمكن أن يكون التربية الفنية هو الحل لمشاكلنا الاجتماعية، لأنه مكان مناسب لرونة لسماع الآراء المختلفة وتقدير وجهات نظر


يميز التربية الفنية عن غيرها من المقررات العلمية، فالتربية الفنية تعطي مساحة للطالب للتعبير عن نفسه و تحترم آرائه الخاصة التي تعبر عن شخصه و محيطه و بيئته، و التي قد تكون متنفس عن الضغوطات او إعطائه إحساس بأهميته



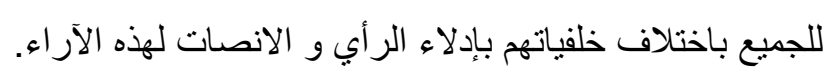


المجتمع السوي الحقيقي هو المجتمع الذي يتحدث فيه الجميع ، ويستمعون إلى بعضهم البعض ، فإن إنتاج الفن ليس شانًَا

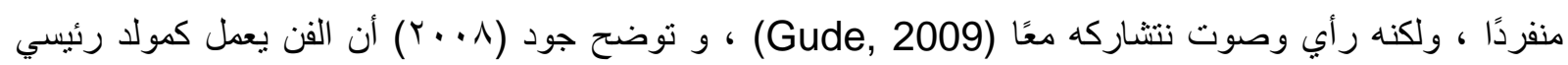

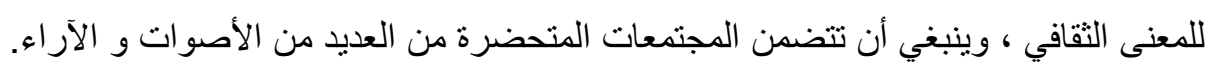

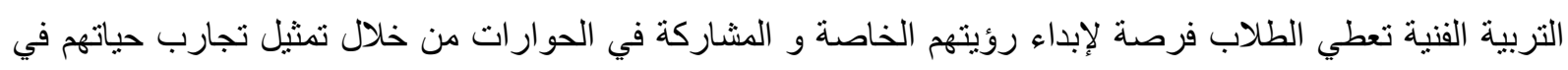

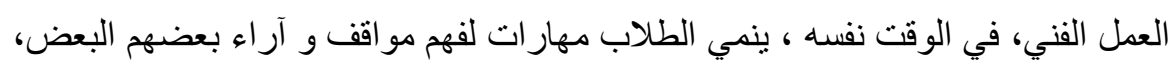

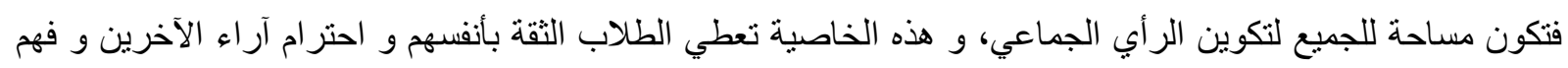
المجتمع الحضاري القائم على التضامن و حرية الرأي للوصول في النهاية إلى حل وسط نهائي بتقاسمه أعضاء



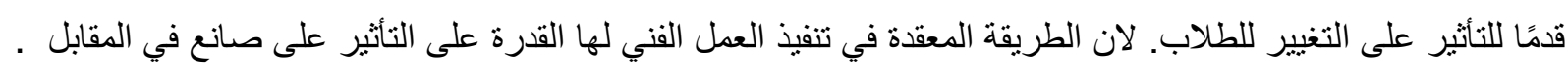

(Dewey, 1934)

لفهم الفن كممارسة حضارية هو فهمه من وجهات نظر متعددة من خلال جو انبه الاجتماعية والثقافية. يمكن استخدام الفن

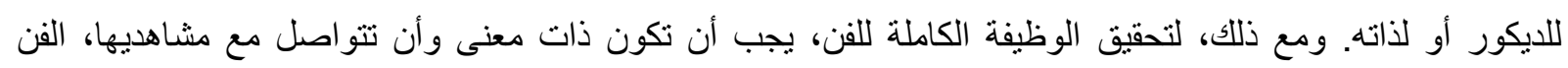
المؤثر له القدرة على حفاظ اعر اف المجتمع أو إلهام التغيير، الجزء الأساسي في تدريس الفن هو ربطه وربطه بـانه بحياتنا الاجنماعية ، لتمكين الطلاب من فهم الفن ووظائفه بشكل كامل (Chalmers (1987) .

الثقافة والتطور الفكري للمجتمع يأكد الباحث الروسي Lev Vygotsky و هو مؤسس نظرية النطور المعرفي الاجتماعي ان العقل الإنساني ليس بمعزل

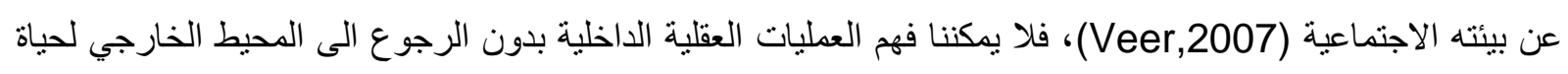
الإنسان ، فمن أجل فهم سلوك الإنسان يجب علينا دراسة الأوضاع و الظروف الخارجية مثل حياته الاجتماعية و الثقافية

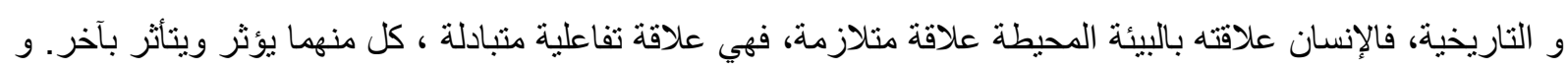

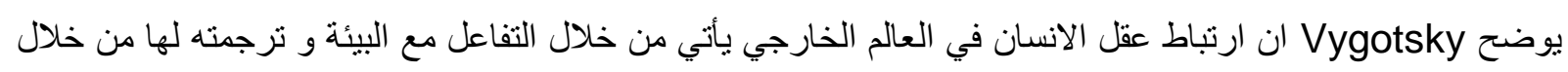
الخبرات المعنوية التي يمر بها . فالمعرفة التي يحصل عليها الانسان هي نتيجة هذه النرجمة ومحاولة

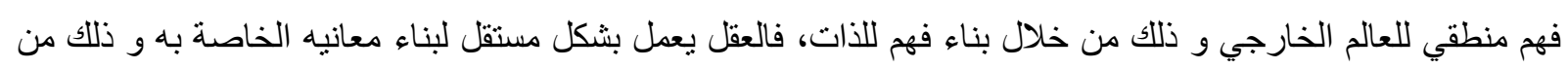
خلال تكوين الآراء و تشكيل الفرضيات، لذلك كل انسان له تصوره الخاص لعالمه، و له وسائله الخاصة لاكتساب المعرفة. من وجهة النظر هذه، يتضح لنا ان كل شعب له أسلوبه و المعاني الخاصة به التي يبنيها لنفسه و ذلك من خلال اعتقاداتهم التي قاموا بتأسيسها من خبر اتهم و تفاعلاتهم مع بيئتهم المحيطة التي هي في تغير مستمر مع الزمن، و و ان كل شعب لـ له


هو المصدر الأولي الذي يزود الأفراد بالمعرفة والمعتقدات والأخلاق التي لها دور أساسي في تكوين المعلومات المعات المعرفية.

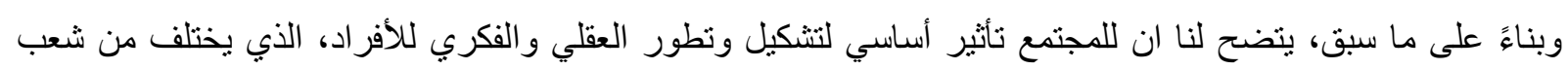
لثعب و الذي قد يحد من الفرص التي يوفر ها للرجل و المرأة (Sivan, 1986) ، فمع مرور الزمن، كل مجتمع يطور



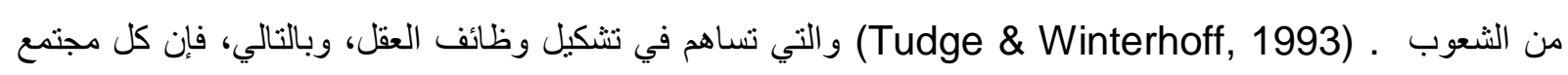
ييني مستويات مختلفة من النظم التنموية، وهذه النظم و السلوكيات معرضة للتغيير و ذلك مع نظوير الأساليب و الوسائل التي يمر بها المجتمع مع مرور التاريخ. 
ساعد تفسير فيجوتسكي فهم التطور المعرفي للإفراد من الناحية الاجتماعية، و فهم الفن كرموز بصرية لمحاولة الانسان

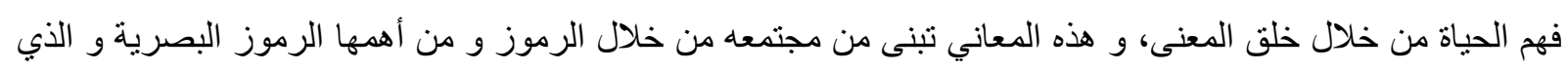
يتوارثها الأبناء للحفاظ على تراثهم أو إلهام التغيير، فهذه الرموز قابلة للتغيير أو التأكيد أو التجديد. فهم أهمية التطور

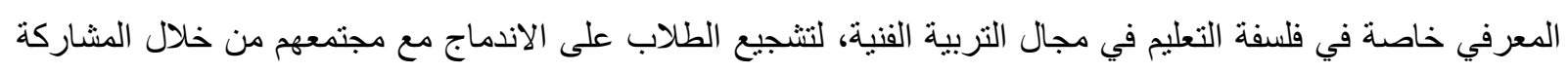

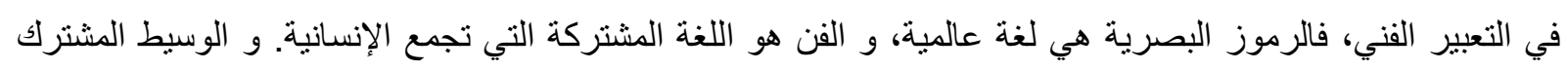
الذي يعزز التو اصل الفعال ولديه القدرة على حل اختلافاتنا (Freedman, 2000).

عرض لنماذج للأعمال المشاركة لتصوير( ( - )
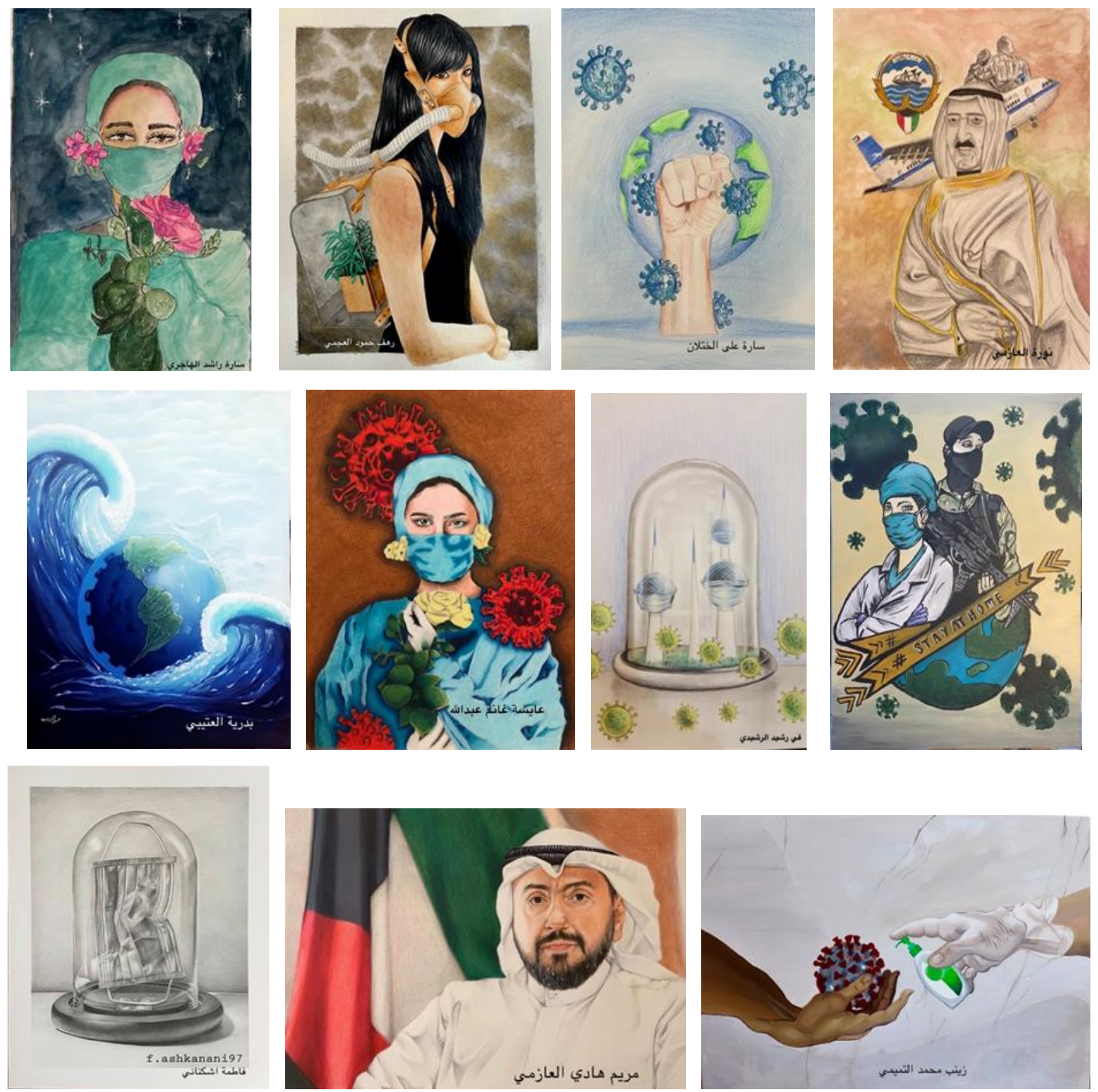
- Anderson, R. L. (2004). Calliope's sisters: A comparative study of philosophies of art (2 ed.).

- Englewood Cliffs, NJ: Prentice Hall.

- Anderson, T., \& Milbrandt, M. K. (2005). Art for life: Authentic instruction in art. New York,

- NY: McGraw-Hill.

- Barone, T. E., \& Eisner, E. W. (2012). Art-based research. Los Angeles, CL: Sage Publications.

- Brookfield, S. (2005). The power of critical theory for adult learning and teaching. Maidenhead,

- Berkshire: Open University Press.

- Chalmers, G. (1987). Culturally based versus universally based understanding of art. In D.

- Blandy, \& K. G. Congdon (Eds.), Art in a democracy (pp.4-12). New York, NY: Teachers College Press.

- Derry, J. (2013). Vygotsky philosophy and education. UK, Oxford: John Wiley \& Sons.

- Dewey, J. (1934). Art as experience. New York, NY: Minton, Balch \& Company.

- Greene, M. (1993). The passions of pluralism multiculturalism and the expanding community.

- Educational Researcher, 22(1), 13-18.

- Gude, O. (2009). Art education for democratic life. Art Education, 62(6), 6-11.

- Gude, O. (2008). Aesthetics making meaning. Studies In Art Education, 50(1), 98-103.

- Sivan, E. (1986). Motivation in social constructivist theory. Educational Psychologist, 21(3),

- 209-233.

- Tudge, J. R., \& Winterhoff, P. A. (1993). Vygotsky, Piaget, and Bandura: Perspectives on the

- relations between the social world and cognitive development. Human Development, 36, 61-61.

- Veer, R. (2007) Vygotsky in context: 1900-1935. In H. Daniels, M. Cole, \& J. V. Wertsch (Eds.), The Cambridge companion to Vygotsky (pp. 21-49). Cambridge, NY: Cambridge University Press.

- Vygotsky, L. (1978). Mind in society: The development of higher psychological processes.

- Cambridge, MA: Harvard University Press. 